\title{
Application of Probability and Mathematical Statistics in Enterprise Risk Analysis
}

\author{
Li Zhang
}

College of Mathematics and Information, China West Normal University, Nanchong, 637002, China

Keywords: Enterprise risk analysis; Probability; Mathematical statistics; Application

\begin{abstract}
In the enterprise risk management process, using the method of probability and mathematical statistics for speculation of relevant data can estimate the risks more conveniently and efficiently, quantitatively analyze the potential risks in the process of enterprise operation and accurately forecast the risk characteristic parameters, so as to get the optimal risk accident avoidance measures, reduce the enterprise operation risks, provide scientific guidance for the enterprise operation, improve the comprehensive competitiveness of enterprises, so that the enterprises can be better developed in the fierce market competition environment. Based on this, the application of probability and mathematical statistics in the enterprise risk analysis is discussed in this paper.
\end{abstract}

\section{Introduction}

An enterprise might face various risks in the process of operation. If the risks cannot be avoided and reduced, the enterprise operation might enter the erroneous zone, causing unnecessary economic loss, and even threatening the future development of the enterprise. Researches prove that the enterprise risks occur mainly because enterprises should face various uncertain factors when making decisions on the operations. As the uncertainty of these factors influence the enterprise operation, in order to reduce the enterprise operation risks, it is necessary to collect all risk information that might influence the decision making, and analyze these uncertain factors and find out relevant laws, so as to achieve the purpose of avoiding risks. In this case, the concept of probability and mathematical statistics is proposed and applied, and the above problems can be effectively solved by using the probability and mathematical statistics, so as to provide scientific guidance for the enterprise decision making.

\section{Brief introduction to probability and mathematical statistics}

The concept of enterprise risk analysis management was proposed in the 1930s, and was developed in the 1950s. At present, risk management has been promoted and applied in various enterprises of some developed countries, and many enterprises specially set up relevant risk management departments and risk management responsible persons to identify, detect and deal with the enterprise operation risks. With the rapid development of the Chinese economy, the reform of market economy has also been continuously deepened, and the autonomous rights of enterprises have been increasing. However, the competition among enterprises also has been gradually fierce, and various uncertain risks faced by the enterprises have been increasingly continuously. In order to reduce the enterprise operation risks, enterprises have used various methods, in which insurance is the most common risk avoidance method in the Chinese enterprises, which is only to deal with the risks by experience and intuition, and is relatively passive to the risk avoidance. Using the method of probability and mathematical statistics for risk management can further improve the enterprise risk avoidance ability.

Probability theory and mathematical theory belong to the mathematical subject, which are mainly used for analysis and research on the random phenomena. Probability and mathematical statistics have special statistical method and concept, with deep contents, and are very closely associated with many fields including economics. In recent years, probability and mathematical statistics have been developed rapidly, and have been widely applied in agriculture, science, military affairs, industry and 
other fields. For example, they are used to analyze the time sequence and forecast the application of space technology in filtering ${ }^{[1]}$. In addition, probability and mathematical statistics have also been gradually applied in the industrial subjects and basic subjects. The key point in this paper is to explore the application of probability and mathematical statistics in the enterprise economic management. Before making the operation decision, enterprises should collect and analyze all risk factors that might influence the decision making, and process and treat the uncertain factors more deeply on the basis of conformance and find out the law hidden.

\section{Application of probability and mathematical statistics in risk forecast}

Enterprise management occurs at random with unexpected characteristics. The economic loss after risk accidents and the influence after economic risks are uncertain. Therefore, it is required to use the method of probability and mathematical statistics to analyze the characteristic value of the risk events and the loss occurring after the risk events. Generally, the common methods include the following:

\section{Interval forecast}

First, assume that in the overall distribution function $f(x, \theta)$, the unknown parameter is $\theta$, the probability of $\hat{\theta}_{1}$ and $\hat{\theta}_{2}$ is given as $1-\alpha$, then $1-\alpha=P\left(\hat{\theta}_{1} \leq \theta \leq \hat{\theta}_{2}\right)$ and the confidence interval of parameter $\theta$ to the confidence probability $1-\alpha$ is a random interval $\left(\hat{\theta}_{1}, \hat{\theta}_{2}\right)$, which is also called the interval estimation of $\theta$, and the accuracy of interval estimation is mainly reflected by the confidence interval, representing the credibility of interval budget probability. $\alpha$ refers to the significance level, mainly reflecting the incredible probability of the interval estimation. During the probability estimation, it is required to carefully analyze the risk data before deciding the interval estimation method of construction. Assume $N\left(\mu, \sigma^{2}\right)$ is the sum of samples, when $\mathrm{n}$ is large enough, the average value of the samples can be taken as random variable distributed by the service normal.

Let $z=(\bar{x}-\mu) / \sigma_{z}$, then $Z$ will follow the random distribution of standard normal distribution, when the sampling errors $\sigma_{z}$ and ${ }^{x}$ are known, it is possible to estimate the interval of the overall average value.

\section{Maximum likelihood estimation method}

Assume that the overall distribution function is known, but there are still several position parameters $\theta$, the values of which cannot be determined, and can be taken in any possible value, so that the sample observation result will generate a large probability, and this value is the estimated value of $\theta$. At this time, $\theta$ can be called the maximum likelihood estimation value of the unknown parameter.

\section{Speculation by known factors}

When estimating the risk probability, enterprises can analyze the distribution of average loss range and the annual loss frequency based on the risk data generated for many years, and calculate the average loss and loss distribution according to these factors. Obviously, the risk accident and amount of loss every year occur separately, so the probability distribution of losses in a year can be speculated ${ }^{[3]}$, and the calculated annual average loss amount is the expected value of risk required, and then enterprises can decide the enterprise operation according to the risk expected value calculated. 


\section{Application of probability and mathematical statistics in enterprise risk analysis}

\section{Case I}

For example, an enterprise wants to produce tablet computer, after comprehensive market investigation, it is found that the supply and demand of tablet computer in the market are the main factors influencing the net investment value of project, while the supply of tablet computer is mainly decided by the number of enterprises. As it is unable to accurate determine how many enterprises will invest in this project, there is certain risk in the net present value of the project investment. In order to establish a more scientific and reasonable investment plan, first it is required to find out the risks that the future net value might face. Therefore, the enterprise owners arrange the marketing department for survey, when the enterprises estimate the future net present value, the condition of enterprises that might participate in the investment and the market probability distribution of tablet computer, they can calculate the distribution of future net value probability of the project. At this time, the risk information of enterprise investment will be gradually defined, by reference to the basic concept of probability, it can be seen that there might be 30-45 enterprises participating in the processing and production of tablet computer in the future, with a net present value of RMB 1.2 million. In the actual decision making, in addition to understanding the distribution of probability information, the managers should also know the influence on the production after risks occur ${ }^{[4]}$. Through the expected value, it is able to reflect the influence of risks on the enterprises more clearly, so as to provide powerful references for the decision makers.

\section{Case II}

An enterprise produces a seasonable product at the cost of RMB 25 per box, with sales price of RMB 70 and profit RMB 45. On the contrary, if the product sales cannot proceed, the enterprise will suffer a loss of RMB 45 per box. After investigating the market sales of the same period, the enterprise concluded the following statistics, as shown in Table 1. At the same time, it applied the sales trend of the current year on this basis, and forecasted the possible sales amount in the current year and established the product production plan in a targeted manner.

Table 1. Sales data of the enterprise in the previous year

\begin{tabular}{cccccc}
\hline Daily sales amount & 100 & 110 & 120 & 130 & Total \\
\hline $\begin{array}{c}\text { Days spent to complete } \\
\text { sales }\end{array}$ & 19 & 37 & 28 & 10 & 90 \\
\hline Probability value & 0.21 & 0.42 & 0.31 & 0.13
\end{tabular}

In the process of enterprise operation, the market demand and market forecast are two main factors influencing the enterprise economic benefit. As the market data are not so complete, during the forecast of market condition, there might be some risks. If the actual demand in the market is 125 boxes, and the enterprise just plans to produce 125 boxes, all goods can be sold, and the enterprise will obtain the best benefit at this time. however, if the actual market demand is less than 125 boxes, for example, the purchase power in the market is only 115 boxes or 120 boxes, there will be the situation of supply exceeding demand, and the product will be unsalable, causing low economic benefit. Therefore, to improve the economic benefit, an enterprise should analyze and calculate the expected income value under each plan, and then take the prevention with the maximum expected income value, which cannot only effectively reduce the decision-making process and avoid unnecessary losses, but can also improve the overall enterprise benefit ${ }^{[5]}$. In the process of analyzing enterprise risks, although the decision makers can analyze the decision-making risks based on the expected value, the expected value is not the only factor influencing the decision making risk, and it is still required to analyze the influence caused by the risks. 


\section{Case III}

A cosmetic sales company takes the TV advertisement and awarding sales as the promotion means, and arranges the sales department to research the two plans in detail and carefully, and it is found that both plans have uncertainty in the increase of sales amount. The distribution of probability is as shown in Table 2.

\begin{tabular}{cccc}
\hline $\begin{array}{c}\text { Increase of sales } \\
\text { amount under the } \\
\text { mode of awarding } \\
\text { sales }\end{array}$ & Probability & $\begin{array}{c}\text { Increase of sales } \\
\text { amount under the } \\
\text { mode of sales } \\
\text { advertisement }\end{array}$ & Probability \\
\hline 1 & $21 \%$ & 5 & $5 \%$ \\
\hline 5 & $21 \%$ & 8 & $35 \%$ \\
\hline 10 & $21 \%$ & 10 & $35 \%$ \\
\hline 15 & $21 \%$ & 15 & $15 \%$ \\
\hline 25 & $21 \%$ & 20 & $10 \%$ \\
\hline
\end{tabular}

According to the calculation formula of expected value, the expected sales amount under the awarding sales mode is calculated as follows:

$E(X)=20 \%+1+20 \% \times 5+20 \% \times 10+20 \% \times 15+20 \% \times 25=11.2(10,000$ bottles $)$

The increase of expected sales amount under TV advertisement is calculated as follows:

$E(X)=5 \%+5+35 \% \times 8+35 \% \times 10+15 \% \times 15+10 \% \times 20=10.8$ (10,000 bottles)

It can be seen from the calculation result that awarding sales generate more sales amount. Therefore, theoretically, the awarding sales method is the optimal. However, in the actual sales, the decision makers are more willing to select TV advertisement to increase the sales amount, and the sales amount increased through TV advertisement is concentrated near the expected value. That is to say, the sales amount 80000 bottles, 100,000 bottles and 150,000 bottles that might increased through TV advertisement approach to the expected value more, and in the actual sales process, the sales probability in this range basically reaches $86 \%$, therefore, using TV advertisement can maintain the sales amount in the expected value more stably. On the contrary, although the awarding sales can generate more efficiency theoretically, in the actual sales, the probability of reaching the expected value is only $65 \%$. Therefore, awarding sales have higher risks and uncertain factors; although the expected value of sales amount through TV advertisement is not higher than that of the awarding sales, its effect is very significant, and it is a more approximate method.

This case better reflects the application of standard deviation concept, as the standard is the square root of variance, while variance is the result of multiplying various possible probabilities by the actual result, there is usually a certain error between the result obtained and the actual condition ${ }^{[5]}$. With the increase of standard deviation, the deviation from the expected value will be more significantly. That is to say, the risk size can be reflected through the standard deviation.

\section{Conclusion}

Overall, many enterprises in China have not deeply realized the risk control and management at present. Although insurance can make up for the losses brought by risks to enterprises to a certain extent, such risk avoidance measures makes the enterprise risk management always in the passive state, which cannot master the risk law, and the enterprise decision makers also cannot manage the risks correctly, and this is unfavorable for the development of enterprises. Therefore, the further deep analysis and promotion of risk management are the top priority in the Chinese enterprises at present. In the process of enterprise risk analysis and forecast, the role of probability and mathematical statistics is very significant. The risks are mainly measured according to the distribution of the possible result probability of the events, while under the risk status, it is possible to guide the decision making by calculating the expected value of risk events, and use the method of standard deviation to measure the risk degree of events with approachable expected value. 


\section{References}

[1] Wang Shuling, Application of Probability Theory and Mathematical Statistics in Economic Life, Scientific and Technology Information, 2009,(21):341-342.

[2] Liao Weiwei, Zhang Wei, Discussion of Applying Probability and Mathematical Statistics for Economic Analysis, North Economics and Trade, 2009,(04):78-79

[3] Xiong Bo, On Application of Mathematical Methods in Economic Research, Contemporary Economics, 2007,(10):154-155.

[4] Wu Yuntian, Status and Role of Mathematical Method in Modern Economics, Journal of Shanxi University of Finance and Economics (higher education edition), 2004,(03):59-60.

[5] Wang Weiping, Li Qian, Brief Discussion on Application of Mathematical Method in Economics, Economist, 2007,(02):83-84.

[6] Wang Xiandong, Chen Rongjun, Research on Example Selection of "Probability Theory” Course of Financial Mathematics, Journal of Changzhou Institute of Technology, 2014,(05):115-119. 